\title{
Compressed Sensing Based on the Single Layer Wavelet Transform for Image Fusion
}

\author{
Guohui Yang, Wude Xu, Bo Zheng, Fanglan Ma, Xuhui Yang, Hongwei Ma, \\ Hongxia Zhang, Genliang Han
}

Institute of Sensing Technology, Gansu Academy of Sciences; Gansu Engineering Research Center of Sensor Technology; Gansu Senor and Application Technology Center, Lanzhou, China

Email: glxygh@163.com

How to cite this paper: Yang, G.H., Xu, W.D., Zheng, B., Ma, F.L., Yang, X.H., Ma, H.W., Zhang, H.X. and Han, G.L. (2016) Compressed Sensing Based on the Single Layer Wavelet Transform for Image Fusion. Journal of Computer and Communications, 4, 107-116.

http://dx.doi.org/10.4236/jcc.2016.415010

Received: October 24, 2016

Accepted: November 25, 2016

Published: November 28, 2016

\begin{abstract}
In this paper, a new method of combination single layer wavelet transform and compressive sensing is proposed for image fusion. In which only measured the high-pass wavelet coefficients of the image but preserved the low-pass wavelet coefficient. Then, fuse the low-pass wavelet coefficients and the measurements of high-pass wavelet coefficient with different schemes. For the reconstruction, by using the minimization of total variation algorithm (TV), high-pass wavelet coefficients could be recovered by the fused measurements. Finally, the fused image could be reconstructed by the inverse wavelet transform. The experiments show the proposed method provides promising fusion performance with a low computational complexity.
\end{abstract}

\section{Keywords}

Image Fusion, Compressed Sensing, Single Layer Wavelet Transform

\section{Introduction}

Image fusion is the technique that integrates complementary and redundant information of multiple images to obtain a composite one, which contains more comprehensive description than any of the individual image. As a result of the processing, the fused image is more useful for human and machine perception or further image processing tasks such as object detection and recognition. By now, many well-known fusion algorithms have been proposed [1]. And the most popular technique up to now is multiresolution decomposition methods, such as pyramid-based methods [2], discrete wavelet transform (DWT)-based methods [3] etc. But most of them need the whole acquisition of the source images, which means that the large of storage burden must be handled especially due to the growing sensor data volumes. 
In recent years, a new technique for simultaneous data sampling and compression known as compressive sensing (CS) [4] [5] [6] [7] has been developed. It builds upon the ground breaking work by Donoho [7] and Candes et al. [8], who showed that under certain conditions, a signal can be precisely recovered from only a small set of measurements. The CS principle provides the potential of dramatic reduction of sampling rates, power consumption and computation complexity in digital data acquisitions. Due to its great practical potentials, it has caused great interest both in academia and industries in the past few years [9] [10]. However, most of existing works in CS remain at the theoretical research.

For image fusion in CS, one natural way is to perform traditional fusion among the reconstructed multiple images after separate recover of each image from the measurements. However, in order to save storage space and reduce the computational complexity, a better method is to directly perform fusion on the measurements, and then to reconstruct the fused image from the fused measurements. Recently, several different fusion strategy based on CS which have been proposed, e.g., a simple maximum selection rule [11] or a self adaptive weighted average fusion scheme based on standard deviation of measurements [12].

In this paper, we proposed an image fusion scheme in a general CS framework. Specifically, we just take single layer wavelet decomposition for image and only measured the high-pass wavelet coefficients of the image with Fast Walsh Hadamard Transform [13], then, fused the low-pass wavelet coefficients and the measurements of high-pass wavelet coefficients with different schemes. The composite coefficients of the fused image are subsequently recovered via the total variation optimization (TV) algorithm [14] and inverse wavelet transform.

This paper is organized as follows. In Section 1, we provide a brief review of CS. The proposed fusion scheme based on CS is described in Section 3. Some experimental results and a discussion are given in Section 4. Finally, Section 5 ends this paper with a conclusion.

\section{Overview of Compressive Sensing}

Consider a real-valued, finite-length, one-dimensional, discrete-time signal $x \in R^{N}$, with elements $x[n] ; n=1,2 \cdots N$. We say that the signal $\mathrm{x}$ is $\mathrm{K}$ sparse if it can be represented as:

$$
x=D \alpha
$$

where $D$ is an $N \times N$ basis matrix and $\alpha$ is an $N \times 1$ vector containing only $\mathrm{K}$ nonzero coefficients. Clearly, $\mathrm{x}$ and $\alpha$ are equivalent representations of the signal, with $\mathrm{x}$ in the time or space domain and $\alpha$ in the $D$ domain. If $K \ll N$, the signal $\mathrm{x}$ is compressible.

In CS, we can not measure or encode $\alpha$ directly, rather, we take the compressive measurements:

$$
y=A x
$$


where $y \in R^{M}$ and $A$ is an $M \times N$ measurement matrix representing the measurement process. Since $M<<N$, the reconstruction of $\mathrm{x}$ from y is generally ill-posed. However, the CS theory is based on the fact that $\mathrm{x}$ has a sparse representation in a known transform domain $D$ (e.g., the DCT and the wavelet). In other words, the transform domain signal $\alpha$ can be recovered perfectly using an optimisation process if $A$ satisfies the restricted isometry property (RIP) [8]. Under some fixed sparsifying basis $D$, such as Gaussian or Bernoulli i.i.d matrices offer universality and optimal performance as measurement matrices [7] [8], but with a high computational complexity. A new sampling operator called Fast Walsh Hadamard Transform [14] is also quite universal but with a lower complexity.

To solve the inverse transform from Equation (2), some non-linear recovery algorithms for such ill-posed problems have been developed [4] [5]-[10]. Unfortunately, most of them require fairly heavy computations in practice. In this paper, we use a new efficient TV minimization scheme based on augmented Lagrangian and alternating direction algorithms, short for "TVAL3 scheme" to solve the problem[14]. It is presented for solving the compressive sensing problem with total variation regularization:

$$
\min _{x} \sum_{i}\left\|D_{i} x\right\| \text {, s.t } \quad A x=\mathrm{y}
$$

where $\mathrm{x}$ is an $N \times 1$ vector, $D_{i} x \in R^{2}$ is the discrete gradient of $\mathrm{x}$ at pixel $\mathrm{i}, A$ is an $M \times N \quad(\mathrm{M}<\mathrm{N})$ measurement matrix, and measurements y are the linear projection of x. $\|$.$\| is 1$-norm(corresponding to the anisotropic TV).

\section{The Proposed Scheme}

\subsection{Sampling}

In order to obtain the measurements of the original images, a type of structured measurement matrices called Fast Walsh Hadamard Transform matrices will be used [14]. The measurement matrices are able to handle the fast computation of matrix-vector multiplication. It is constructed by the Hadamard Matrix. The Hadamard matrix of dimension $2^{k}$ for $\mathrm{k}$ are given by the recursive formula:

$$
\begin{gathered}
H_{0}=[1] \\
H_{1}=\frac{1}{\sqrt{2}}\left[\begin{array}{cc}
1 & 1 \\
1 & -1
\end{array}\right]
\end{gathered}
$$

And in general

$$
H_{K}=\frac{1}{\sqrt{2}}\left[\begin{array}{cc}
H_{K-1} & H_{K-1} \\
H_{K-1} & -H_{K-1}
\end{array}\right]
$$

This is also known as the Hadamard-ordered Walsh Hadamard matrix. Other orders, such as sequency order, dyadic order, and so on can be obtained by reordering the rows of the Hadamard matrix. Walsh Hadamard matrices 'in various orders have recently received growing concern due to the extensive application in engineering field.

To achieve the Fast Walsh Hadamard transform matrix, it is necessary to understand the "so-called Kronecker product". 
For any two matrices $A=\left[a_{i j}\right]_{p \times q}$ and $B=\left[b_{i j}\right]_{r \times l}$, the Kronecker Product of two matrices is defined as:

$$
A \otimes B=\left[\begin{array}{cccc}
a_{11} B & a_{12} B & \cdots & a_{1 q} B \\
a_{21} B & a_{22} B & \cdots & a_{2 q} B \\
\vdots & \vdots & \vdots & \vdots \\
a_{p 1} B & a_{p 2} B & a_{p 3} B & a_{p 4} B
\end{array}\right]
$$

In order to better understand the meaning of the Kronecker product, we define two new operators vec and mtx. The vec operator is to make all the columns of a matrix into a vector, and $\mathrm{mtx}$ is the inverse operator of vec that separates the vector into several equal-length vectors and forms a matrix.

According to "Kronecker product" theorem, Matrix $A \in R^{m \times n}$ can constructed by the Kronecker product formula:

$$
A=\left(A_{1} \otimes A_{2}\right)
$$

where $A_{1} \in R^{(m / p) \times(n / q)}$ and $A_{2} \in R^{p \times q}$, m and $\mathrm{n}$ are chosen to satisfy that $\mathrm{m}$ and $\mathrm{n}$ are divisible by $\mathrm{p}$ and $\mathrm{q}$, respectively. Then matrix-vector multiplication can be computed by:

$$
\begin{aligned}
& A x=\operatorname{vec}\left(A_{1} m t x(x) A_{2}^{T}\right. \\
& A^{T} y=\operatorname{vec}\left(A_{2}^{T} m t x(y) A_{1}\right)
\end{aligned}
$$

Detailed derivation process in [14]. Using the Kronecker product, the Formula (4) can rewritten as:

$$
H_{K}=H_{1} \otimes H_{K-1}
$$

For any two vector $\mathrm{x}$ with the length of $2^{k}$, denote $x=\left[\begin{array}{ll}x_{1}^{T} & x_{2}^{T}\end{array}\right]$, where $x_{1}$ and $x_{2}$ are the same size. The Hadamard-ordered Walsh Hadamard transform $\left(W H T_{h}\right)$ can be expressed as:

$$
H_{K} x=\left(H_{1} \otimes H_{K-1}\right) x
$$

Due to the Basic KP theorem, it follows

$$
\begin{aligned}
H_{K} x= & \operatorname{vec}\left(H_{K-1} m t x(x) H_{1}^{T}\right) \\
& =\operatorname{vec}\left(H_{K-1}\left[\begin{array}{ll}
x_{1} & x_{2}
\end{array}\right] H_{1}^{T}\right) \\
& =\operatorname{vec}\left(\left[\begin{array}{ll}
H_{K-1} x_{2} & H_{K-2} x_{2}
\end{array}\right]\right) H_{1}^{T} \\
& =\frac{1}{\sqrt{2}} \operatorname{vec}\left(\left[\begin{array}{ll}
H_{K-1} x_{1} & H_{K-1} x_{2} \\
1 & 1 \\
1 & -1
\end{array}\right]\right) \\
& =\frac{1}{\sqrt{2}} \operatorname{vec}\left(\left[\begin{array}{ll}
H_{K-1} x_{1}+H_{K-1} x_{2} & H_{K-1} x_{1}-H_{K-1} x_{2}
\end{array}\right]\right) \\
& =\frac{1}{\sqrt{2}}\left[\begin{array}{l}
H_{K-1} x_{1}+H_{K-1} x_{2} \\
H_{K-1} x_{1}-H_{K-1} x_{2}
\end{array}\right]
\end{aligned}
$$

A implementation of the fast $W H T_{h}$ according to recursive Formula (10) would only have a computational complexity of $O(N \log N)$, but it is $O\left(N^{2}\right)$ of a general $W H T_{h}$. We can see that only additions and subtractions are involved while implementing the fast $W H T_{h}$.

Let $x_{i}$ represent the vectorized signal of the $\mathrm{i}$-th wavelet sub-band. The corres- 
ponding measurement output vector can be written as:

$$
y_{i}=A x_{i}, \quad i=1,2,3
$$

In each wavelet sub-band, we set the same sampling rate, so the number of measurements for each sub-band are the same.

\subsection{Fusion}

Multi-resolution wavelet decomposition has a noticeable advantage in the representation of a signal. In our fusion scheme, we take a single-level CDF-97 wavelet transform to decompose the original image into approximation coefficients and the detail coefficients. High frequency sub-band include the main energy of the original image and can considered to be sparse, but the scale coefficients low frequency sub-band is not considered to be sparse. So the low-frequency and high frequency coefficients multiplied with measurement matrix A together will destroyed the correlation between low-frequency approximate weight coefficient and the detail coefficients and lead to a poor reconstruction results. Hence, we preserving the low-pass wavelet coefficients and only measured the high-pass wavelet coefficients of the image. Then we fuse them with different fusion rules.

\section{A) Fusion rule I}

Let $A^{K}$ and $B^{K}$ be the $\mathrm{k}$-th $(\mathrm{K}=1,2,3)$ low-frequency sub-images of image $\mathrm{A}$ and image $B$ respectively. The fused approximation coefficient of the $k$-th low-frequency sub-images $F^{K}$ can be formulated as:

$$
F^{K}=A^{K}+B^{K}-\frac{\overline{A^{K}}+\overline{B^{K}}}{2}
$$

where $\overline{A^{K}}$ and $B^{K}$ are the mean value of $A^{K}$ and $B^{K}$. This strategy can keep the low-frequency better compare with general average weighted fusion strategy.

\section{B) Fusion rule I}

Image structural similarity (SSIM) [15] is a kind of effective image quality evaluation index based on the hypothesis that the human visual perception is highly adapted for extracting structural information from a scene. Inspired by this, we use SSIM to extract the structure relation between two high-frequency sub-images measurement.

The structural similarity (SSIM) is designed by modeling any image distortion as the combination of structure comparison function $s(x, y)$, luminance comparison function $l(x, y)$, contrast comparison function $c(x, y)$. SSIM is defined as:

$$
\begin{gathered}
\sigma_{x}^{2}=\sqrt{\frac{1}{M N-1} \sum_{i=1}^{M} \sum_{j=1}^{N}\left(x(i, j)-\mu_{x}\right)^{2}} \\
\sigma_{x y}^{2}=\frac{1}{M N-1} \sum_{i=1}^{M} \sum_{j=1}^{N}\left(x(i, j)-\mu_{x}\right)\left(y(i, j)-\mu_{y}\right) \\
l(x, y)=\frac{2 \mu_{x} \mu_{y}+C_{1}}{\mu_{x}^{2}+\mu_{y}^{2}+C_{1}} \\
c(x, y)=\frac{2 \sigma_{x} \sigma_{y}+C_{2}}{\sigma_{x}^{2}+\sigma_{y}^{2}+C_{2}}
\end{gathered}
$$




$$
\begin{gathered}
s(x, y)=\frac{\sigma_{x} \sigma_{y}+C_{3}}{\sigma_{x y}+C_{3}} \\
\operatorname{SSIM}(x, y)=\frac{\left(2 \mu_{x} \mu_{y}+C_{1}\right)\left(2 \sigma_{x y}+C_{2}\right)}{\left(\mu_{x}^{2}+\mu_{y}^{2}+C_{1}\right)\left(\sigma_{x}^{2}+\sigma_{y}^{2}+C_{2}\right)} \\
=l(x, y) c(x, y) s(x, y)
\end{gathered}
$$

where $\mu_{x}$ and $\mu_{y}$ are the mean, $\sigma_{x}, \sigma_{y}$ and $\sigma_{x y}$ are the standard deviation and covariance, respectively. All of these is computed on the high-frequency sub-images measurement between image $\mathrm{x}$ and $\mathrm{y}$. constant $C_{1}, C_{2}, C_{3}$ are included to avoid instability when denominator is very close to zero. Specifically, $C_{1}=\left(K_{1} L\right)^{2}$, $C_{2}=\left(K_{2} L\right)^{2}$ where $\mathrm{L}$ is the dynamic range of the high-frequency sub-images measurement(maximum for two corresponding high frequency sub-band image measurement), and $K_{1}<<1, K_{2}<<1$ is a small constant, in our experiments we set the parameter at 0.05 , the other parameters of SSIM are all in accordance with Ref. [15].

For image fusion, it is useful to apply the SSIM locally rather than globally. In our experiments $\mu_{x}, \sigma_{x}$ and $\sigma_{x y}$ are computed within a local $2 \times 2$ square window, which moves pixel-by-pixel over the entire image. At each step, the local statistics and SSIM are calculated within the local window.

At last, the mean SSIM (MSSIM) index to measure the similarity of two images defined as follows:

$$
\operatorname{MSSIM}(x, y)=\frac{1}{M} \sum_{j=1}^{M} \operatorname{SSIM}\left(x_{j}, y_{j}\right)
$$

where $x_{j}$ and $y_{j}$ are the image information at the $\mathrm{j}$-th local window, and $\mathrm{M}$ is the number of local windows of the image.

For the detail coefficients fusion, we let $G A^{K}$ and $G B^{K}$ be the measurement of the $\mathrm{k}$-th $(\mathrm{K}=1,2,3)$ high-frequency sub-images of image $\mathrm{x}$ and image $\mathrm{y}$ respectively. Then the fused high-frequency sub-image measurement $G F^{K}$ can be written as:

$$
G F^{K}=G A^{K}+G B^{K}-\operatorname{MSSIM}(x, y)\left(G A^{K}+G B^{K}\right) / 2
$$

where $\operatorname{MSSIM}(x, y)$ is the mean structural similarity index between two corresponding high frequency sub-band image measurement.

Consequently the fused high-frequency coefficient can be obtained through the TV minimization scheme based on augmented Lagrangian and alternating direction algorithms (TVAL3), see [14]. The specific steps of proposed method are summarized in Algorithm 1.

Algorithm 1. Image fusion in compressed sensing.

1. Perform single layer wavelet transform for input Images;

2. Fuse the low-frequency wavelet coefficient by fusion rules I and measured each high-pass wavelet sub-band coefficients with measurement matrix $\mathrm{WHT}_{h}$;

3. Fuse each high-pass measurement by fusion rules II;

4. Reconstruct fused high-pass wavelet coefficient via the TVAL3 algorithm;

5. Recover the fused image via inverse wavelet transform. 


\section{C) Algorithm}

We recover the fused high-frequency coefficient from the fused high-frequency measurement using the TVAL3 algorithm [14].

\section{Experimental Results and Performance Evaluation}

In this section, three groups of test images are employed for the performance evaluation to illustrate the effectiveness of the proposed approach. Three pairs of source images including computed tomography (CT) and magnetic resonance imaging (MRI) images shown in Figure 1(a) and Figure 1(b), infrared and visual images, see Figure 2(a) and Figure 2(b), and optical multi-focus images, shown in Figure 3(a) and Figure 3(b). For more information about the images, refer to [16]. The high-frequency sampling rate is set at 0.2 , and the total number of recover data is $40 \%$ of all the data. The sampling rate of other two methods is also set to 0.4 All experiments are implemented on an Intel Core(TM)2 Duo $2.33 \mathrm{GHz}$ computer, and the simulation software is MATLAB 7.0.1.

In our experiment, We compare the proposed method with method in [11], which uses a "double-star" sampling pattern and a maximum absolute value selection fusion

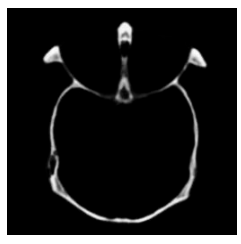

(a)

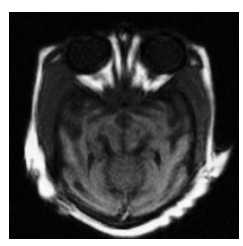

(b)

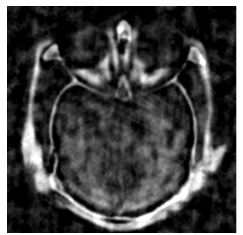

(c)

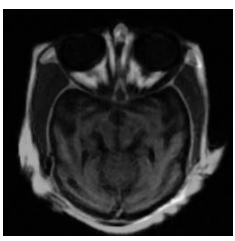

(d)

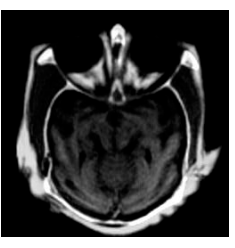

(e)

Figure 1. (a)original CT image; (b) original MRI image; (c) fused image by CS-MS; (d) fused image by CS-SD; (e) fused images of the proposed method.

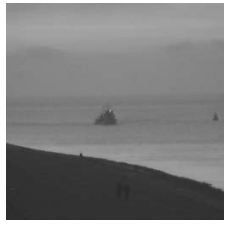

(a)

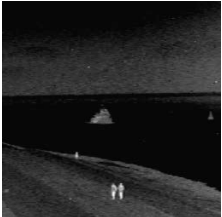

(b)

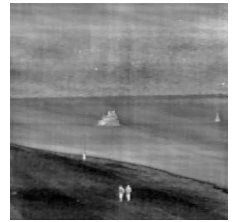

(c)

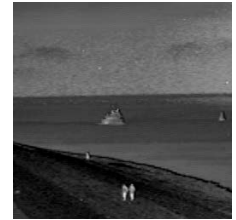

(d)

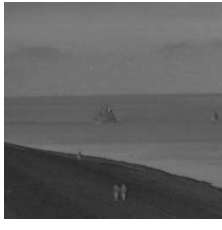

(e)

Figure 2. (a) original visible image; (b) original infrared image; (c) fused image by CS-MS; (d) fused image by CS-SD; (e) fused images of the proposed method.

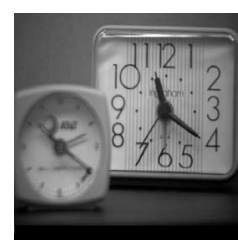

(a)

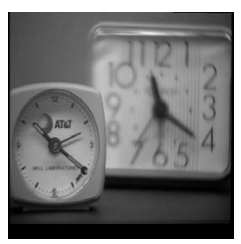

(b)

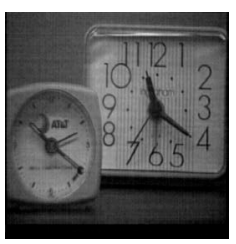

(c)

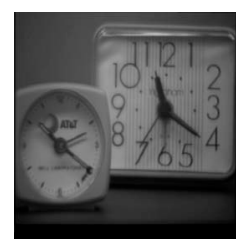

(d)

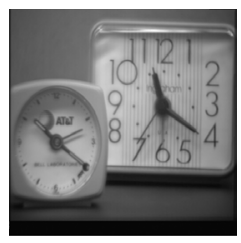

(e)

Figure 3. (a) original right focus image; (b) original left focus image; (c) fused image by CS-MS; (d) fused image by CS-SD; (e) fused images of the proposed method. 
rule (CS_MS) and [12], which uses a "star-shaped" sampling mode and a weighted average fusion rule based on Standard deviation (CS-SD). In medical image fusion, It's easy to see that the fusion results of the proposed method has a higher contrast and contains most of the details of the individual input images than CS_MS and CS-SD. Especially, the CS-SD has a low-contrast and contains noise compared with the original images. For the IR and visual images fusion, we can see that the results of our method also exhibit the best visual quality. Compared with our method, CS_MS scheme is not suitable for infrared and visual images fusion due to the distortion of brightness contrast and contain much strip noise. For the CS-SD, it losses contrast in the goals. In the last groups, our methods in the multifocus images fusion have a equal visual quality compared with CS-SD and is better than CS_MS.

For further comparison, several objective criteria are used to evaluate the fusion results. The first criterion is the average gradient (AG), which is commonly used to evaluate the clarity of image, the greater AG is, the sharper is the image. The next two criterion is the mutual information (MI) and edges keep degrees $\left(Q^{A B} / F\right)$, MI reflects the total amount of information that the fused image contains that of original image and $Q^{A B} / F$ considers the amount of edge information transferred from the input images to the fused images. For the two criteria, the larger the value is, the better is the fusion result. Finally, we give the CPU running time for fusion and CS reconstruction, respectively.

The performance evaluation of fusion results for three methods is shown in Table 1 . It is obvious that our proposed method outperforms the other two methods in terms of MI and Q, which explain that the fused image of our method contains more detail and edge information compare with those of the other methods. It is consistent with the visual effect. Though the average gradient (AG) in the med image fusion is a little bigger than our method, its visual effect is far worse than ours. At last, it's worth noting that the CPU running time of our method for fusion and CS reconstruction is far less than the other two methods. In general, from the subjective visual comparison, and the objective index comparison, we can come to a conclusion that the proposed method gets a better performance than the other two methods.

Table 1. Performance evaluation of fusion results for our proposed method and other schemes.

\begin{tabular}{cccccc}
\hline Image & Method & AG & MI & Q & T (s) \\
\hline \multirow{2}{*}{ Med } & CS-MAV & $\mathbf{8 . 6 7}$ & 1.56 & 0.51 & 583 \\
$256 * 256$ & CS-SD & 8.06 & 3.74 & 0.50 & 547 \\
& Ours & 8.02 & 3.93 & 0.77 & 3 \\
Military & CS-MAV & 3.65 & 2.03 & 0.39 & 468 \\
$256 * 256$ & CS-SD & 1.21 & 3.34 & 0.26 & 364 \\
& Ours & 4.01 & 3.42 & 0.49 & 3 \\
Clock & CS-MAV & 3.57 & 5.22 & 0.46 & 7280 \\
$512 * 512$ & CS-SD & 1.97 & 6.75 & 0.52 & 7269 \\
& Ours & 4.35 & 6.79 & 0.57 & 19 \\
\hline
\end{tabular}




\section{Conclusions}

In the paper, we put forward a kind of effective image fusion scheme based on compressive measurements. Compare with traditional method, we firstly take single layer wavelet transform for original image. Then, according to the characteristics of high frequency coefficients sparse, we only measured the high-pass wavelet coefficients with a low sampling rate. At last, we fuse low-frequency wavelet coefficient and highfrequency measurement with different fusion rules and recovery them with a new efficient TV minimization algorithm. Simulation results indicate that proposed scheme provides efficiency and promising fusion results. In addition, owing to the proposed scheme only needs incomplete measurements rather than acquiring all the samples of the whole image, the computational complexity significantly reduces. Moreover, because of using Fast Walsh Hadamard Transform, the CPU running time of our method for fusion and CS reconstruction is far less than other two methods.

CS image fusion is still in the stage of exploration, many problems remain to solve (e.g., the relationship between the original image and measurements and how to design the best fusion rule). Therefore the study of new and more advanced fusion rules matched with CS principle is another research topic in our future work.

\section{Acknowledgements}

This work was supported by the Gansu Academy of Sciences Youth Foundation under Grant 2015-QN-06. The associate editor coordinating the review of this manuscript and approving it for publication was Dr. Bo Zheng. The authors are with the Institute of sensing technology, Gansu Academy of Sciences (e-mail: glxygh@163.com; zhengboem@163.com).

\section{References}

[1] Smith, M.I. and Heather, J.P. (2005) Review of Image Fusion Technology in 2005. Proceedings on Defense and Security Symposium, Orlando, FL, 28 March-1 April.

[2] Liu, Z., Tsukada, K., Hanasaki, K., Ho, Y.K. and Dai, Y.P. (2001) Image Fusion by Using Steerable Pyramid. Pattern Recogn. Lett., 22, 929-939.

http://dx.doi.org/10.1016/S0167-8655(01)00047-2

[3] Wang, H., Peng, J. and Wu, W. (2002) Fusion Algorithm for Multisensor Images Based on Discrete Multiwavelet Transform. IEE Proc. Vis. Image Signal Process., 149, 283-289. http://dx.doi.org/10.1049/ip-vis:20020612

[4] Baraniuk, R.G. (2007) Compressive Sensing. IEEE Signal Process. Mag., 24, 118-120, 124. http://dx.doi.org/10.1109/MSP.2007.4286571

[5] Candes, E.J. and Wakin, M.B. (2008) An Introduction to Compressive Sampling. IEEE Signal Process. Mag., 25, 21-30. http://dx.doi.org/10.1109/MSP.2007.914731

[6] Romberg, J. (2008) Imaging via Compressive Sampling. IEEE Signal Process. Mag., 25, 14-20. http://dx.doi.org/10.1109/MSP.2007.914729

[7] Donoho, D.L. (2006) Compressed Sensing. IEEE Trans. Inform. Theory, 52, 1289-1306. http://dx.doi.org/10.1109/TIT.2006.871582

[8] Candes, E., Romberg, J. and Tao, T. (2006) Robust Uncertainty Principles: Exact Signal Re- 
construction from Highly Incomplete Frequency Information. IEEE Trans. Inform. Theory, 52, 489-509. http://dx.doi.org/10.1109/TIT.2005.862083

[9] Tsaig, Y. and Donoho, D.L. (2006) Extensions of Compressed Sensing. Signal Processing, 86, 533-548. http://dx.doi.org/10.1016/j.sigpro.2005.05.028

[10] Donoho, D.L., Tsaig, Y., Drori, I. and Starck, J.-L. (2006) Sparse Solution of Underdetermined Linear Equations by Stagewise Orthogonal Matching Pursuit. Mar., preprint.

[11] Wan, T., Canagarajah, N. and Achim, A. (2008) Compressive Image Fusion. In: Proceeding of 15 th IEEE International Conference on Image Processing, San Diego, California, U.S.A, IEEE, 1308-1311.

[12] Li, X. and Qin, S.-Y. (2011) Efficient Fusion for Infrared and Visible Images Based on Compressive Sensing Principle. IET Image Process., 5, 141-147.

[13] Cen, Y.-G., Chen, X.-F., Cen, L.-H. and Chen, S.-M. (2010) Compressed Sensing Based on the Single Layer Wavelet Transform for Image Processing. Journal on Communications, 31, No. 8A.

[14] Li, C.B. (2009) An Efficient Algorithm For Total Variation Regularization with Applications to the Single Pixel Camera and Compressive Sensing. Master of Arts, Rice University, Houston, Texas.

[15] Wang, Z., Bovik, A.C., Sheikh, H.R. and Simoncelli, E.P. (2004) Image Quality Assessment: From Error Visibility to Structural Similarity. IEEE Trans. Image Process., 13, 600-612. http://dx.doi.org/10.1109/TIP.2003.819861

[16] http://www.imagefusion.org

Submit or recommend next manuscript to SCIRP and we will provide best service for you:

Accepting pre-submission inquiries through Email, Facebook, LinkedIn, Twitter, etc.

A wide selection of journals (inclusive of 9 subjects, more than 200 journals)

Providing 24-hour high-quality service

User-friendly online submission system

Fair and swift peer-review system

Efficient typesetting and proofreading procedure

Display of the result of downloads and visits, as well as the number of cited articles

Maximum dissemination of your research work

Submit your manuscript at: http://papersubmission.scirp.org/

Orcontact jcc@scirp.org 